\title{
The Optimization of the Multi-Atmospheric Ar-Xe Laser
}

\author{
S. W. A. Gielkens, W. J. Witteman, V. N. Tskhai, and P. J. M. Peters
}

\begin{abstract}
The quasi-steady-state conditions of the multiatmospheric $e$-beam sustained Ar-Xe laser are investigated. It is observed that the duration of the stationary period depends on the $e$-beam current, discharge power deposition, and gas pressure. The laser efficiency can be as high as $8 \%$. Beyond the stationary period the efficiency drops. The pulse energy with optimum efficiency depends strongly on the gas pressure. The maximum discharge efficiency of $5 \%-6 \%$ is at high pressure not sensitive to the input power. The best results are obtained for 4 bar with a discharge input power of $8 \mathrm{MW} / \ell$. The pulse duration with corresponding output energies is $12 \mu \mathrm{s}$ with $10 \mathrm{~J} / \ell$ and $16 \mu \mathrm{s}$ with $16 \mathrm{~J} / \ell$ for $e$-beam currents of 0.4 and $0.9 \mathrm{~A} / \mathrm{cm}^{2}$, respectively. An analysis of the quasi-steady-state conditions that include the effects of electron collision mixing and atomic quenching is presented. The effects of output power saturation by the fractional ionization and atomic collisions are in agreement with the observations. The analysis clarifies the optimum performance conditions.
\end{abstract}

Index Terms - Electric discharge pumping, electron beam pumping, gas lasers, lasers, laser thermal factors, power lasers, pulsed lasers.

\section{INTRODUCTION}

$\mathbf{T}$ HE STUDY of the laser transitions between the $5 d$ and $6 p$ bands of xenon [1]-[7] is of considerable interest for several reasons. First of all, it is from a scientific point of view remarkable that these lasing infrared transitions can be very efficient up to $8 \%$ depending on discharge conditions and that pulse energies up to $15 \mathrm{~J} / \ell$ with power densities of several MW/ $\ell$ can be obtained [7]. Secondly, the apparent favorable kinetic chain of this laser process based on threebody collisions challenged the development of CW systems with output powers in the order of watts [8], [9]. This became successful with RF excitation of a mixture at 90 torr in narrow waveguide structures where output power densities of about $0.27 \mathrm{~W} / \mathrm{cm}^{3}$ were obtained, which is two or three orders of magnitude higher than what was previously known for lowpressure atomic discharge Xe lasers. This breakthrough in the gas laser development of obtaining high power combined with the typical high optical quality opens the gate to many new promising applications, e.g., the field of remote sensing and communications. The high efficiency and high output power

Manuscript received July 17, 1997; revised October 9, 1997. These investigations in the program of the Foundation for Fundamental Research on Matter were supported in part by the Netherlands Technology Foundation (STW).

The authors are with the Department of Applied Physics, University of Twente, 7500 AE Enschede, The Netherlands.

Publisher Item Identifier S 0018-9197(98)01097-5. make this laser also a competitor for the well-developed $\mathrm{CO}_{2}$ laser. The advantages of this new atomic laser are the absence of dissociation and the regeneration of the laser gas, and the much shorter wavelength of about $2 \mu \mathrm{m}$ compared to $\mathrm{CO}_{2}$ lasers.

The full exploration of the new system requires a detailed study of its parameters and its kinetic chain of the inversion production. The understanding of the laser and the quantitative information on the kinetics can then be used for the design of an optimized system. From an experimental point of view, it is attractive to study this atomic xenon laser by means of an $e$-beam sustained device because this technique has shown so far to be most productive and efficient for radiation production. Moreover, it allows to follow the effects of discharge parameters and gas composition more or less independently and in this way unravels kinetic processes that otherwise may be strongly mixed. For instance, the e-beam produces a stable homogeneous plasma, independent on the gas pressure, so that the study of gas density effects is not hampered by plasma instabilities that in self-sustained discharges are automatically introduced by the increase of the gas pressure. In principle, the $e$-beam sustained discharge allows to follow more or less independently the effects of discharge current, $e$-beam current, and gas pressure.

In our previous work [7], we used a short $e$-beam pulse of only $1.2 \mu \mathrm{s}$ and a much longer discharge pulse. These experiments showed the fast drop of the output power after termination of the $e$-beam. This work clarified the necessity of simultaneous operation of discharge and $e$-beam. Furthermore, the experiments revealed the more or less quadratic dependence of the optimum input power on the gas pressure. The experiments also brought forward the question to what extent we are dealing with the quasi-steady state during the simultaneous presence of the pulses and what the saturation mechanisms are. We particularly want to have more insight into the quenching effects of electrons and atoms. To study these questions, we reconstructed our system to have simultaneous pulses for the $e$-beam and sustainer of about $20 \mu \mathrm{s}$. For this device, we observed the output waveforms as a function of e-beam current, discharge current, and gas pressure. A kinetic model is developed to get more insight into the kinetic processes.

\section{EXPERIMENTAL SETUP}

The electron gun is based on a plasma cathode and is described elsewhere [10]. The $e$-beam current density after 
passing the $15-\mu \mathrm{m}$-thick Ti foil was varied between 0.25 and $0.9 \mathrm{~A} / \mathrm{cm}^{2}$. In our experiments, the accelerator voltage was kept constant at $185 \mathrm{kV}$. The discharge circuit consists of three capacitors with a capacitance of $30 \mu \mathrm{F}$ each and two inductors each of $400 \mathrm{nH}$ to provide for a more or less rectangular shape with a duration of about $20 \mu \mathrm{s}$. The discharge is switched on by the $e$-beam. The discharge is maintained between the foil and an additional electrode. To avoid sputtering, we used the foil as anode. When this foil was used as cathode the sputtering resulted already in foil rupture at a current of $20 \mathrm{kA}$. The resonator consists of a flat totally reflecting $\mathrm{Cu}$ mirror and a plan-parallel $\mathrm{ZnSe}$ output coupler with a reflectance of $50 \%$. These mirrors are separated by $90 \mathrm{~cm}$. The distance between the electrodes is $2 \mathrm{~cm}$ and the cross section of the $e$-beam is $3 \times 53 \mathrm{~cm}^{2}$. The laser extraction volume is $0.31 \ell$ and the base vacuum in the laser chamber was $5 \times 10^{-9}$ bar. Highpurity argon (99.9990\%) and xenon (99.990\%) were used. The beam and discharge current were measured by Rogowski coils. The accelerating and discharge voltage were measured by resistive voltage dividers. The contribution of an inductive element to the measured voltage appeared to be negligible. By multiplication of the measured discharge current and voltage the input power of the discharge was calculated. The power deposition by the $e$-beam is calculated from stopping power data [11]. The laser oscillates on several transitions between the $5 d$ and $6 p$ levels of Xe. The temporal profile of the total output power is measured by a fast uncooled InAs photodiode (EG\&G J12-18c) in combination with a CdTe window that transmits all laser lines but blocks visible radiation. The total output energy is detected by a pyroelectric joulemeter (Gentec ED 500). By comparison of the measured energy with the measured waveform detected by the photodiode, the amplitude of this diode signal is converted into units of power.

\section{EXPERIMENTAL OBSERVATIONS}

The typical behavior of the pulsed experiment is the appearance of the output pulse shortly after the onset of the discharge pulse, followed by a quasi-steady-state regime where the e-beam current, discharge current, and output power are more or less constant and finally the region with the premature fall-off of the output pulse whereas the discharge and $e$-beam pulses are still present (see Fig. 1). The stationary period is then determined by the time during which the output power is more or less constant. At the end of this period, we always observe a continuous fast drop of the output. The present paper only considers the total lasing potential of the two bands by investigation of the multiwave mode. We always observe that the total laser output of the oscillator with broad-band reflectors does not show any substantial modulation during the stationary period whereas the observed individual lines are strongly modulated during this period which is due to the well-known line competition. The experiments give us the stationary duration of the output as a function of pressure, discharge power, and $e$-beam current. In the following, we will show various quantities as a function of the discharge power density for $e$-beam current densities of 0.4 and $0.9 \mathrm{~A} / \mathrm{cm}^{2}$. The dissipated $e$-beam power is not simply a constant times the

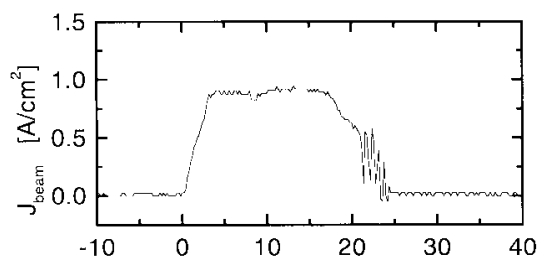

(a)

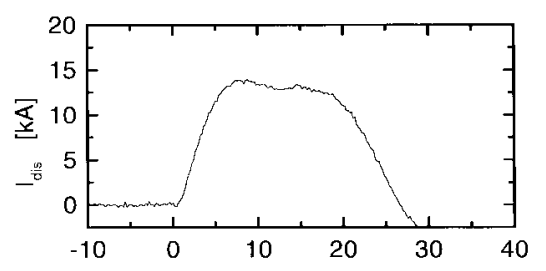

(b)

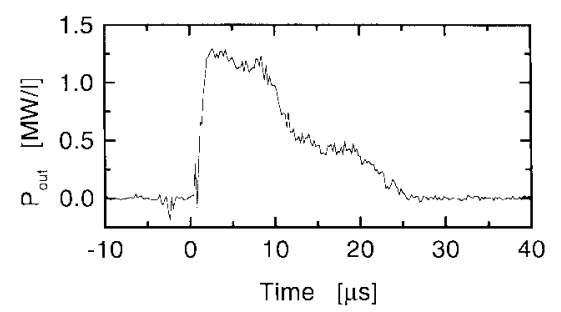

(c)

Fig. 1. Temporal profiles of the (a) beam current density $J_{\text {beam }}$, (b) discharge current $I_{\text {dis }}$, and (c) laser output power $P_{\text {out }}$.

product of the chosen $e$-beam current and gas pressure because at higher input powers of the discharge when the stationary period is short, it is observed that a substantial part of the stationary output power is already present during the build-up time of the $e$-beam. For each picture, the total input power can be inferred from Fig. 2. In this figure for each experimental condition the corresponding beam input power and discharge power is plotted. The results of the stationary time as a function of the discharge power are shown in Fig. 3(a) and (b) for $e^{-}$ beam current densities of 0.4 and $0.9 \mathrm{~A} / \mathrm{cm}^{2}$, respectively. It is clearly seen that the stationary time strongly depends on pressure. Although the experimental data are somewhat scattered owing to experimental fluctuations, the stationary time is roughly inversely proportional to the discharge power.

Beyond this stationary regime, the output power and laser efficiency decrease. The output power in the stationary regime as a function of discharge power is plotted in Fig. 4(a) and (b) for e-beam current densities of 0.4 and $0.9 \mathrm{~A} / \mathrm{cm}^{2}$, respectively. The general behavior of increase of the output power is, up to a value that depends on the gas pressure, proportional to the discharge power. The higher the gas pressure the larger the value of the discharge power that limits this proportionality regime. The intrinsic efficiency of the laser with respect to the discharge power is plotted in Fig. 5(a) and (b) for $e$-beam current densities of 0.4 and $0.9 \mathrm{~A} / \mathrm{cm}^{2}$, respectively. These values are calculated from the ratio of the total output power minus the output power generated by the $e$-beam only and the discharge power. The total efficiency is determined by the ratio of the output power and total input power. When we plot the total efficiency versus the total input 


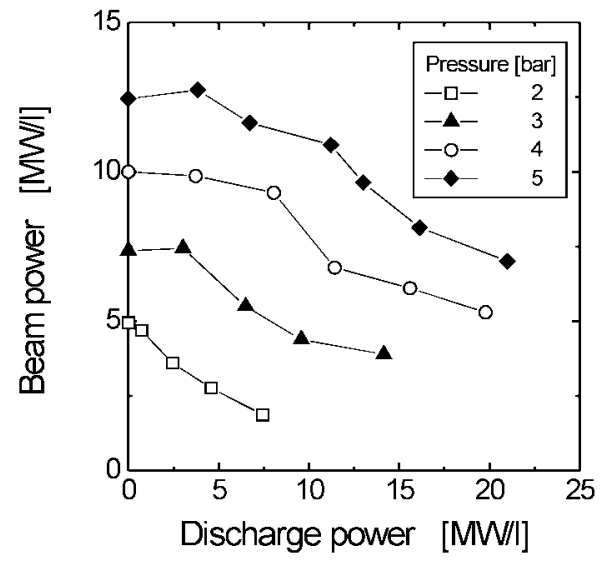

(a)

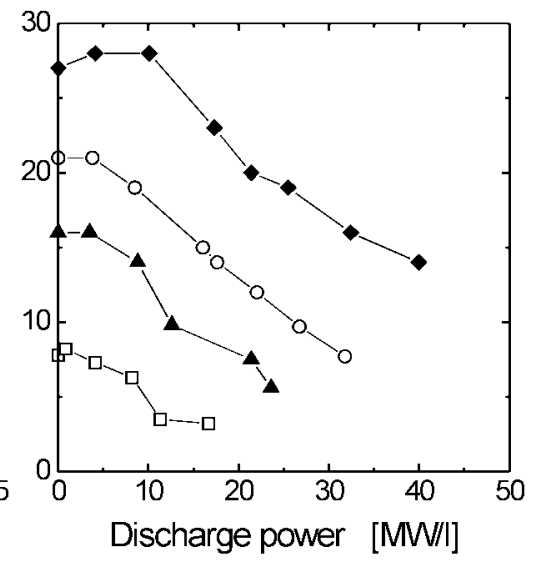

(b)

Fig. 2. For each experimental condition, the beam input power that corresponds to the given discharge power is plotted.

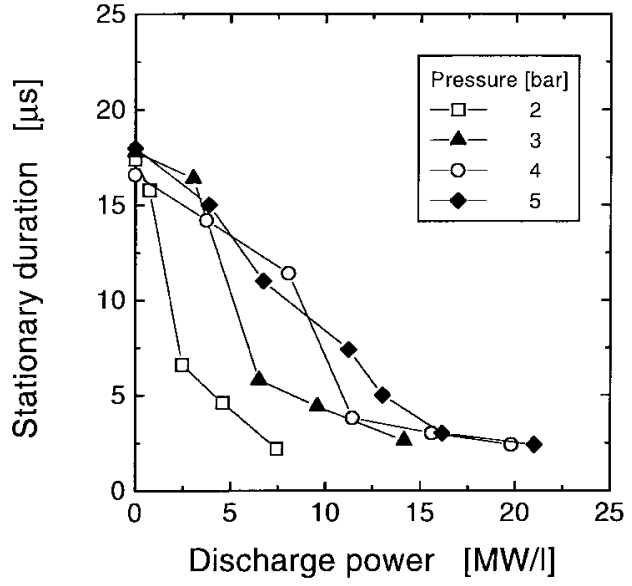

(a)

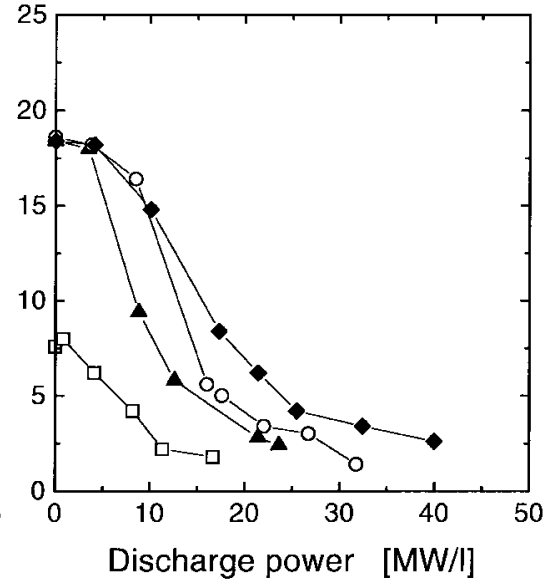

(b)

Fig. 3. The duration of the quasi-steady-state behavior of the laser as a function of the discharge power at $e$-beam current densities of (a) 0.4 and (b) $0.9 \mathrm{~A} / \mathrm{cm}^{2}$.

power, as is done in Fig. 6(a) and (b) for e-beam current densities of 0.4 and $0.9 \mathrm{~A} / \mathrm{cm}^{2}$, we see that the maximum efficiency drops with total input power and that the highest efficiency of about $8 \%$ is reached for input powers below 10 $\mathrm{MW} / \ell$ at a low gas pressure. For each input power, the gas pressure can be optimized and the optimized pressure increases with input power. Finally we plotted the available output energy per pulse during the stationary period as a function of discharge power and total power (see Fig. 7). It is remarkable that this output energy is sensitive to the $e$-beam current and that it has a maximum around 4 bar.

\section{ANALYSIS OF THE SySTEM}

We shall discuss a kinetic model of the laser process by means of a flow diagram of the kinetics shown in Fig. 8. It indicates the main species and kinetic reactions that are typically expected for the e-beam sustained multi-atmospheric Ar-Xe laser with only $0.5 \% \mathrm{Xe}$. The electron beam mainly ionizes the argon gas proportional to its density [Ar] so that the ion production by the $e$-beam can be written as $c_{e} J_{\text {beam }}[\mathrm{Ar}]$ where $c_{e}$ is a constant and $J_{\text {beam }}$ the beam current density. These ions are lost by three-body collisions with $\mathrm{Ar}$ to form $\mathrm{Ar}_{2}^{+}$. The main process suffered by the molecular argon ions is the formation of $\mathrm{ArXe}^{+}$in collisions with Xe. The recombination of $\mathrm{ArXe}^{+}$leads to the formation of the higher excited states of $\mathrm{Xe}$, which are subsequently quenched by atomic collisions to reach the upper laser level $5 d$ manifold. The lower laser level $6 p$ manifold is at multiatmospheric pressure mainly quenched by Ar to reach the metastable $6 s^{\prime}$ level. There is also some radiative decay to the metastable $6 s$ level. Then, the metastable Xe atoms will produce $\mathrm{ArXe}^{*}$ excimers in three-body collisions with $\mathrm{Ar}$. These excimers decay by radiative dissociation and form again the ground state. It is seen that the relevant xenon levels above the metastable levels are separated by about $1 \mathrm{eV}$, an energy comparable with the average electron energy of the discharge. For that reason, it is generally accepted that the discharge mainly contributes to the excitation and ionization from the metastable level. Because of the low xenon content the contribution of the $e$-beam to the formation rate of $\mathrm{Xe}^{+}$ is negligible compared to the discharge contribution. In this 


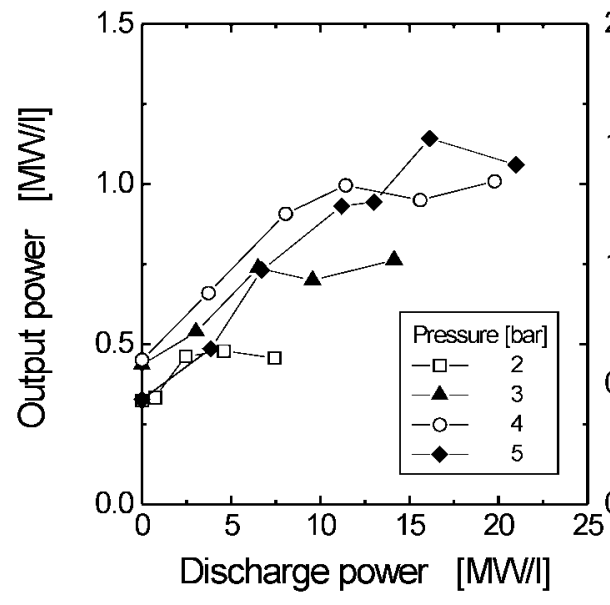

(a)

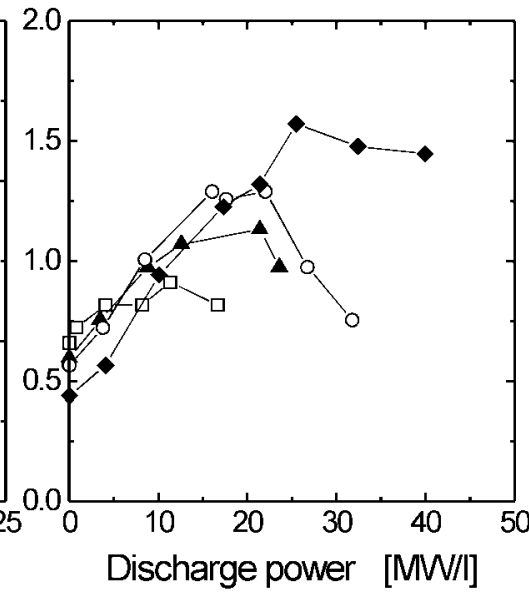

(b)

Fig. 4. The output power density during the stationary duration as a function of the discharge power for an $e$-beam with a current density of (a) $0.4 \mathrm{~A} / \mathrm{cm}^{2}$ and (b) $0.9 \mathrm{~A} / \mathrm{cm}^{2}$.

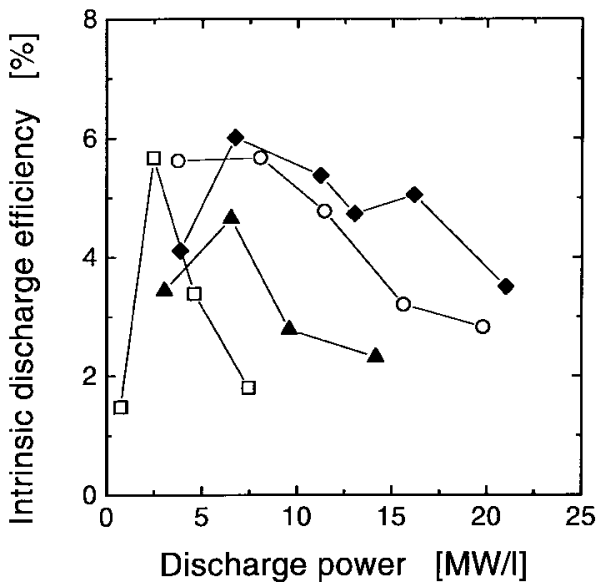

(a)

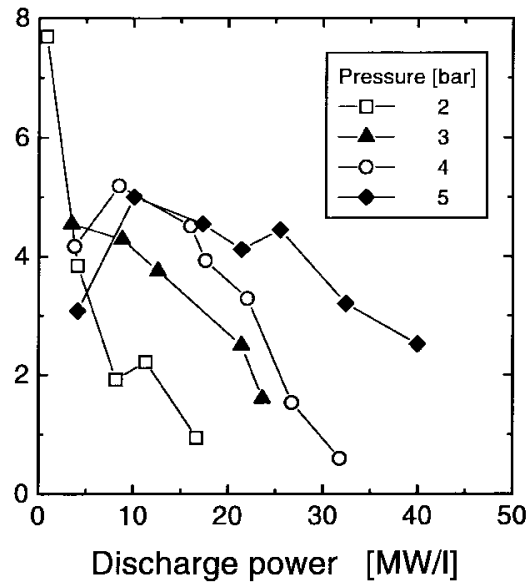

(b)

Fig. 5. The intrinsic discharge efficiency versus the discharge input power density during the stationary time of the laser for (a) 0.4 A/cm ${ }^{2}$ and (b) 0.9 $\mathrm{A} / \mathrm{cm}^{2}$. This efficiency is defined by the ratio of the total output power minus the output power generated by $e$-beam pumping alone and the discharge power.

way, the discharge is effective in producing $\mathrm{Xe}^{+}$which in three-body collisions is converted into $\mathrm{ArXe}^{+}$. In principle, the kinetic chain of the discharge and laser process forms a closed cycle bounded by the metastable and ionization level of $\mathrm{Xe}$. In this steady-state process, the supply of metastable Xe atoms by the $e$-beam compensates for the above-mentioned loss of metastable atoms that decay via the excimer to the ground state. It is experimentally observed that after an early termination of the $e$-beam the discharge impedance increases drastically and the output drops.

The electrons are delivered by both the $e$-beam and discharge. The discharge conditions depend on the $e$-beam current density. In the case of pure $e$-beam pumping the average electron energy increases slightly with increasing $e$-beam current density. At lower $e$-beam current and constant discharge power the reduced electric field strength $(E / N)$, the drift velocity, and average energy of discharge electrons are higher and the electron density is lower. Apart from the formation kinetics of the laser inversion which depends strongly on the discharge parameters and gas pressure, there is also considerable quenching of the inversion by collisional mixing of the $6 p$ and $5 d$ manifolds by both electrons and atoms. For that reason, the laser performance is a strong interplay of discharge power, e-beam current, and gas pressure; each parameter can be optimized in relation with the other ones.

\section{KINETICS}

Below, we will describe a kinetic model for the steady-state behavior of the laser. The rate equation for $\mathrm{Ar}^{+}$is mainly given by the following process:

$$
\frac{d\left[\mathrm{Ar}^{+}\right]}{d t}=c_{e} J_{\text {beam }}[\mathrm{Ar}]-k_{1}\left[\mathrm{Ar}^{+}\right][\mathrm{Ar}]^{2}
$$

where $c_{e}$ is a constant of proportionality, $J_{\text {beam }}$ is the $e$ beam current density, and $k_{1}$ is the formation constant of 


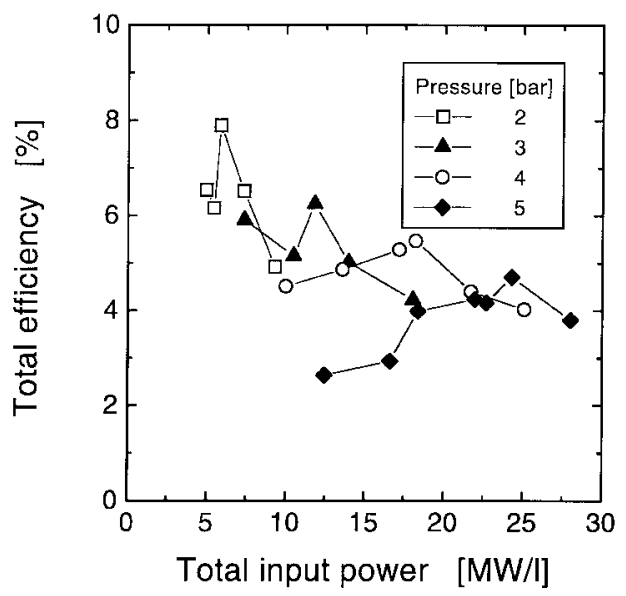

(a)

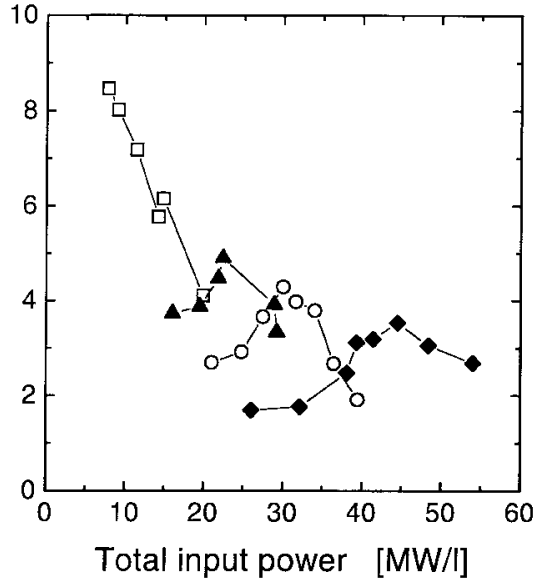

(b)

Fig. 6. The total efficiency as a function of the total input power density for (a) $0.4 \mathrm{~A} / \mathrm{cm}^{2}$ and (b) $0.9 \mathrm{~A} / \mathrm{cm}^{2}$. This efficiency is defined by the ratio of the total output power and the total input power.

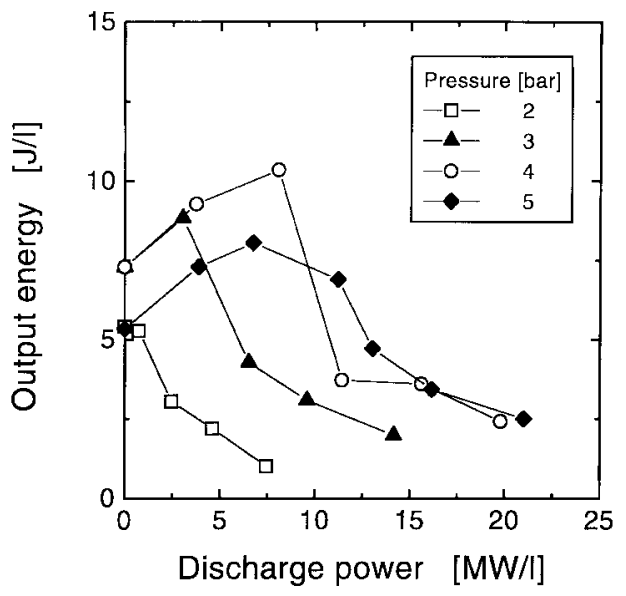

(a)

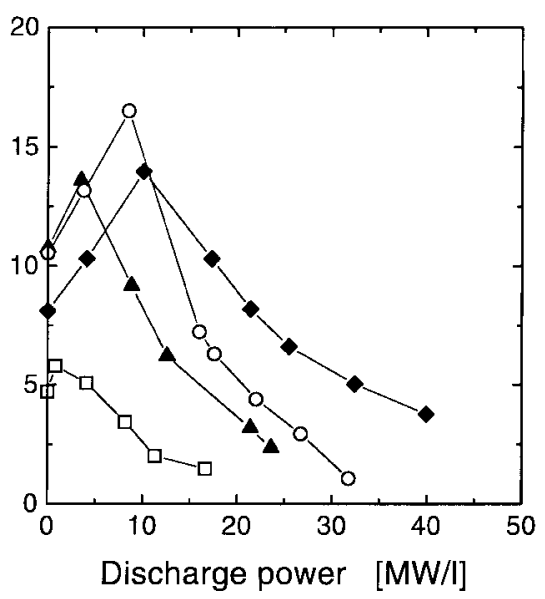

(b)

Fig. 7. The dependence of the output energy on the discharge power density during the stationary operation of the laser for (a) $0.4 \mathrm{~A} / \mathrm{cm}^{2}$ and (b) 0.9 $\mathrm{A} / \mathrm{cm}^{2}$. Notice that this energy also peaks as a function of the pressure.

molecular argon ions. The main process for $\mathrm{Ar}_{2}^{+}$in our system is described by

$$
\frac{d\left[\mathrm{Ar}_{2}^{+}\right]}{d t}=k_{1}\left[\mathrm{Ar}^{+}\right][\mathrm{Ar}]^{2}-k_{2}\left[\mathrm{Ar}_{2}^{+}\right][\mathrm{Xe}]
$$

where $k_{2}$ is the formation constant for molecular $\mathrm{ArXe}^{+}$. The molecular $\mathrm{ArXe}^{+}$formation in the e-beam chain is described by

$$
\frac{d\left[\mathrm{ArXe}^{+}\right]}{d t}=k_{2}\left[\mathrm{Ar}_{2}^{+}\right][\mathrm{Xe}]-k_{e}\left[\mathrm{ArXe}^{+}\right] n_{e}
$$

where $k_{e}$ is the recombination rate constant of $\mathrm{ArXe}^{+}$and $n_{e}$ is the electron density. The main kinetic chain for $\mathrm{Xe}^{+}$ is dominated by the discharge power density $P_{d}$ that ionizes the metastable Xe atoms, and by its quenching by three-body collisions to form $\mathrm{ArXe}^{+}$. Since in the model the discharge will ionize the metastable xenon atoms, we take the production rate proportional to the discharge power

$$
\frac{d\left[\mathrm{Xe}^{+}\right]}{d t}=c_{d} P_{d}-k_{3}\left[\mathrm{Xe}^{+}\right][\mathrm{Ar}]^{2}
$$

where $c_{d}$ is a constant of proportionality and $k_{3}$ is the rate constant for formation of $\mathrm{ArXe}^{+}$. The molecular $\mathrm{ArXe}^{+}$ formation in the discharge chain is then given by

$$
\frac{d\left[\mathrm{ArXe}^{+}\right]}{d t}=k_{3}\left[\mathrm{Xe}^{+}\right][\mathrm{Ar}]^{2}-k_{e}\left[\mathrm{ArXe}^{+}\right] n_{e} .
$$

The xenon metastables are produced by both the $e$-beam and discharge. The production by the e-beam is in the stationary state equal to the ionization rate of Ar. Similarly, in the discharge chain the production rate of the metastables is in our model equal to the ionization rate of $\mathrm{Xe}$, which means that the discharge has no effect on the density of the metastables:

$$
\frac{d\left[\mathrm{Xe}^{*}\right]}{d t}=c_{e} J_{\text {beam }}[\mathrm{Ar}]-k_{4}\left[\mathrm{Xe}^{*}\right][\mathrm{Ar}]^{2}
$$




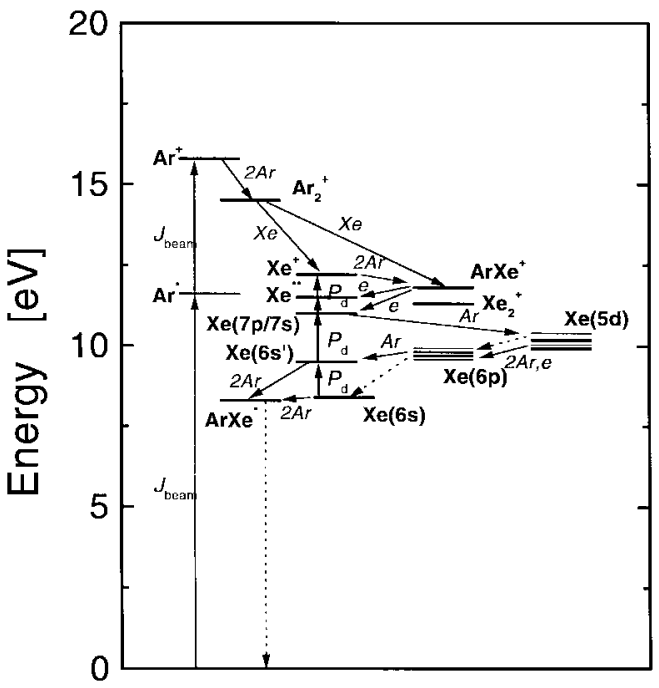

Fig. 8. Scheme of the most important kinetic reactions and species for the plasma chemistry of the $\mathrm{Ar}$-Xe laser pumped by an e-beam sustained discharge. The bold names are labels of the energy levels, whereas the italic ones are the particles involved in reactions indicated by a solid arrow. The dotted arrows denote radiative transitions.

where $k_{4}$ is the rate constant for three-body quenching of the metastables. For the stationary state the electron density $n_{e}$ is equal to the sum of the ions, which can be deduced from (1)-(5)

$$
\begin{aligned}
n_{e}= & c_{e} J_{\text {beam }}\left(\frac{1}{k_{1}[\mathrm{Ar}]}+\frac{[\mathrm{Ar}]}{k_{2}[\mathrm{Xe}]}\right)+\frac{c_{d} P_{d}}{k_{3}[\mathrm{Ar}]^{2}} \\
& +\frac{1}{k_{e} n_{e}}\left(c_{e} J_{\text {beam }}[\mathrm{Ar}]+c_{d} P_{d}\right) .
\end{aligned}
$$

The electron density is plotted in Fig. 9 as a function of the discharge power for various pressures. The values of the various constants are given in Table I. The values for $k_{1}, k_{2}, k_{3}, k_{4}$, and $k_{e}$ have been taken from [1]. The value for $c_{e}$ has been calculated from the beam input power and the $W$ value for $\mathrm{Ar}^{+}$[2]. The value of $c_{d}$ has been chosen such that the model fits the laser output power as a function of the discharge power (see the next section). It is seen that $n_{e}$ is not sensitive to the gas pressure. The metastable density becomes according to (6)

$$
\left[\mathrm{Xe}^{*}\right]=\frac{c_{e} J_{\text {beam }}}{k_{4}[\mathrm{Ar}]} .
$$

\section{LASER PROCESS}

In the following, we shall describe the main kinetics of the laser process. The inversion is dominated on the one hand by the excitation processes of the $e$-beam and discharge. The production rate of the upper laser level with density $n_{2}$ will be in our model proportional to the ionization rate of argon i.e. $c_{e} J_{\text {beam }}[\mathrm{Ar}]$. Since the ionization rate of xenon is taken proportional to the discharge power, the production rate of the upper laser level is in our model also proportional to the discharge power $\left(\alpha P_{d}\right)$. On the other hand, the inversion is quenched by electrons and atoms. The lasing transitions have high oscillator strengths so that they are tightly coupled

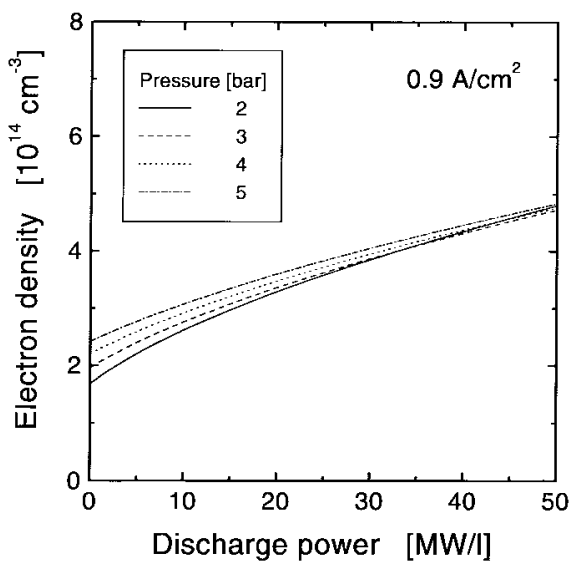

Fig. 9. Electron density versus the discharge power density at various pressures as calculated from (7).

TABLE I

Values of the Reaction Constants Optimized to Match Most Closely the EXPERIMENTAL Results AT AN $e$-BEAm CuRRENT Density of $0.9 \mathrm{~A} / \mathrm{cm}^{2}$

\begin{tabular}{c|c|c||c|c|c}
\hline Constant & Value & Unit & Constant & Value & Unit \\
\hline \hline$k_{1}$ & $2.3(-31)$ & $\mathrm{cm}^{6} / \mathrm{s}$ & $p_{1}$ & $2.0(-12)$ & $\mathrm{cm}^{3 / \mathrm{s}}$ \\
\hline$k_{2}$ & $2.0(-10)$ & $\mathrm{cm}^{3} / \mathrm{s}$ & $p_{2}$ & $3.7(-32)$ & $\mathrm{cm}^{6} / \mathrm{s}$ \\
\hline$k_{3}$ & $2.0(-31)$ & $\mathrm{cm}^{6} / \mathrm{s}$ & $q_{0}$ & $1.35(-6)$ & $\mathrm{cm}^{2} \mathrm{~A}^{1 / 2} / \mathrm{s}$ \\
\hline$k_{4}$ & $6.7(-34)$ & $\mathrm{cm}^{6} / \mathrm{s}$ & $r$ & $2.2(-11)$ & $\mathrm{cm}^{3 / \mathrm{s}}$ \\
\hline$k_{\mathrm{e}}$ & $1.6(-7)$ & $\mathrm{cm}^{3} / \mathrm{s}$ & $\alpha$ & $3.1(18)$ & $1 / \mathrm{J}$ \\
\hline$c_{\mathrm{e}}$ & 65 & $\mathrm{~cm}^{2} / \mathrm{As}$ & $\beta$ & $2.2(18)$ & $1 / \mathrm{J}$ \\
\hline$c_{\mathrm{d}}$ & $5.0(17)$ & $1 / \mathrm{J}$ & & & \\
\hline
\end{tabular}

by electron collisions. The electron collisions strive for thermalization of the $6 p$ and $5 d$ manifolds characterized by the electron temperature. The quenching of the upper laser level by the electrons is proportional to its density as well as the electron density

$$
\left(\frac{d n_{2}}{d t}\right)_{e}=-q n_{e} n_{2}
$$

where $q$ is a constant of proportionality. The quenching parameter $q$ depends on the plasma conditions like the average electron energy. At higher average energy the collision frequency increases and consequently the quenching rate too. It is expected that the higher the plasma conductivity determined by the e-beam current, the lower the average energy of the electrons and the smaller the quenching parameter. The parameter $q$ will then depend on the $e$-beam current density. In order to get a reasonable fit with the experimental observations, we approximate $q$ with the relation $q=q_{0} / I^{1 / 2}$.

Similarly the quenching by two- and three-body-collisions of the atoms is given by

$$
\left(\frac{d n_{2}}{d t}\right)_{a}=-p_{1} N n_{2}-p_{2} N^{2} n_{2}
$$




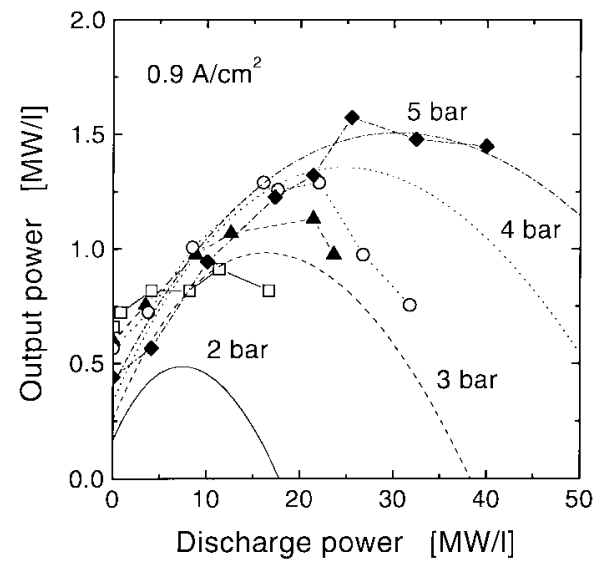

Fig. 10. Curves of the output power as a function of the discharge power at an $e$-beam current density of $0.9 \mathrm{~A} / \mathrm{cm}^{2}$. For various pressures, these are calculated from (16) with values of the constants as mentioned in Table I. The symbols in this figure coincide with those in Fig. 4(b).

where $p_{1}$ and $p_{2}$ are proportionality constants and $N$ the gas density practically equal to [Ar]. Including the stimulated emission, the upper laser level density is given by

$$
\begin{aligned}
\frac{d n_{2}}{d t}= & \alpha P_{d}+c_{e} J_{\text {beam }} N-\rho B\left(n_{2}-n_{1}\right) \\
& -q n_{e} n_{2}-p_{1} N n_{2}-p_{2} N^{2} n_{2}
\end{aligned}
$$

where $\rho$ is the radiation density and $B$ the Einstein coefficient for stimulated emission. Since the mentioned quenching processes strive for thermalization between the laser levels, we write for the lower laser level with density $n_{1}$

$$
\begin{aligned}
\frac{d n_{1}}{d t}= & \beta P_{d}+\rho B\left(n_{2}-n_{1}\right)+q n_{e} n_{2} \\
& +p_{1} N n_{2}+p_{2} N^{2} n_{2}-r N n_{1}
\end{aligned}
$$

where $\beta P_{d}$ is the pumping of the lower level and $r N n_{1}$ the quenching of the lower level, which is proportional to the gas density. To maintain the inversion, $r$ is much larger than $p_{1}$ and the decay of the lower laser level by three-body collisions is negligible.

The radiation production $W$ is equal to

$$
W=\rho B\left(n_{2}-n_{1}\right) h v \text {. }
$$

The inversion is given by

$$
n_{2}-n_{1}=\frac{1}{B h v \tau_{\text {cav }}}
$$

where $\tau_{\text {cav }}$ is the decay time of the resonator determined by its quality factor. For our system we find $n_{2} \sim n_{1}$. With this we obtain by adding (11) and (12) in the stationary regime

$$
c_{e} J_{\text {beam }} N+P_{d}(\alpha+\beta)=r N n_{2} .
$$

Substituting (15) into (11) and eliminating $\left(n_{2}-n_{1}\right)$ by (13), we obtain

$$
\begin{aligned}
W= & h v\left(\alpha P_{d}+c_{e} J_{\text {beam }} N\right) \\
& -h v\left\{c_{e} J_{\text {beam }} N+P_{d}(\alpha+\beta)\right\} \\
& \cdot\left(\frac{q}{r} \frac{n_{e}}{N}+\frac{p_{1}}{r}+\frac{p_{2} N}{r}\right) .
\end{aligned}
$$

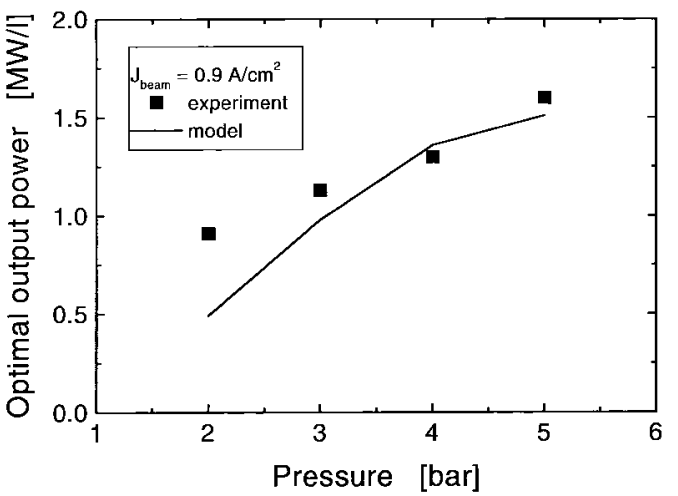

Fig. 11. Plot of the optimal value of the output power density versus the pressure for the data of Fig. 12. Experimental values are indicated by symbols, whereas the calculated points are depicted by the curve.

The initial values for the parameters $p_{1}, p_{2}, q_{0}$, and $r$ are based on [2]. Together with $\alpha$ and $\beta$ the final values of these parameters are obtained by matching the calculated curves with the measured data. These values are listed in Table I. The output power according to (16) together with our experimental data are plotted in Fig. 10 for an $e$-beam current density of $0.9 \mathrm{~A} / \mathrm{cm}^{2}$. In Fig. 11, the optimal output power is plotted versus the pressure for both the experimental data and the calculated curves. These figures show that a reasonable agreement with the experiments can be accomplished by this model. Studying this result, we make the following conclusions.

1) For small input power of the sustainer, the output power scales proportionally. In this regime, $n_{e}$ is low enough for quenching to be negligible. In (16), this is expressed by the term containing $n_{e} / N$, which is often referred to by fractional ionization.

2) The output saturates with the discharge power which is due to the fact that $n_{e}$ increases with the discharge power, as seen in Fig. 9, and finally outweighs the pumping term. The calculated results are shown in Fig. 10.

3) The electron quenching depends on the fractional ionization $n_{e} / N$. The electron density is not sensitive to the gas density according to (7) and shown in Fig. 9. This means that according to (16) the maximum obtainable power increases strongly with the gas pressure, which is in agreement with the observations plotted in Fig. 4. It is also seen in Figs. 5 and 6 that the efficiency for the same input power at larger input powers increases with the gas pressure which can also be explained by the decreasing fractional ionization with increasing gas pressure.

4) From (16), it is predicted that atomic quenching is negligible up to about 3 bar, whereas the strong electron quenching decreases more or less inversely with the gas pressure. This means that the output power and the efficiency will have a maximum value that depends on both the discharge power (or total power) and gas pressure. According to Fig. 5, the maximum efficiency before saturation as a function of discharge power is 


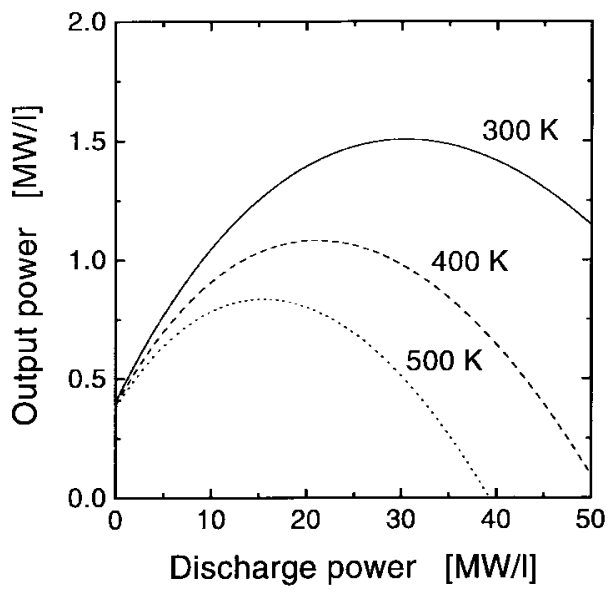

(a)

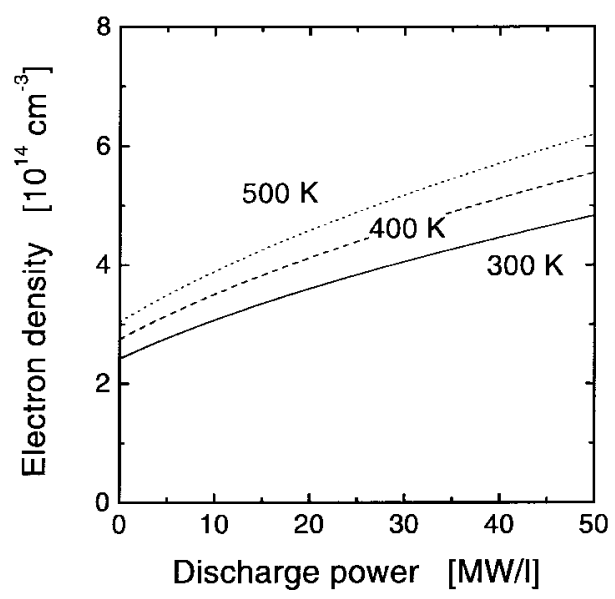

(b)

Fig. 12. The dependence of (a) the output power density and (b) the electron density on the discharge power density at various temperatures. Note the dramatic drop of the output power at high input powers, though the electron density increases only slightly.

attained at about 4 bar for both $e$-beam current densities. However, since the discharge pumping power at which the output power is optimal is proportional to the pressure (Fig. 4), one can expect that the maximum power may still increase with densities above 5 bar.

\section{TEMPERATURE EFFECT ON THE Kinetics}

From our analysis, it can also be deduced that the output is sensitive to the gas temperature because the rate constants of ion-electron recombination and the three-body collisions in the kinetic chain depend on the temperature. It is found that the higher the temperature, the higher the electron density so that the losses by quenching increase and the output power drops. The rate constants of the three-body ion formation, $k_{1}$ and $k_{3}$, have a gas temperature dependence proportional to $T_{g}^{-3 / 2}$, whereas the dissociative rate constant $k_{e}$ has a temperature dependence proportional to $1-\exp \left(-\varepsilon_{v} / k T_{g}\right)$, where $\varepsilon_{v}$ is the fundamental vibrational energy [12]. The temperature dependence of $k_{e}$ may be well approximated by $T_{g}^{-1}$, because $\varepsilon_{v}$ is only about $6 \mathrm{meV}$ [13]. Substituting these dependences into (7), we see for increasing temperature an increase of $n_{e}$

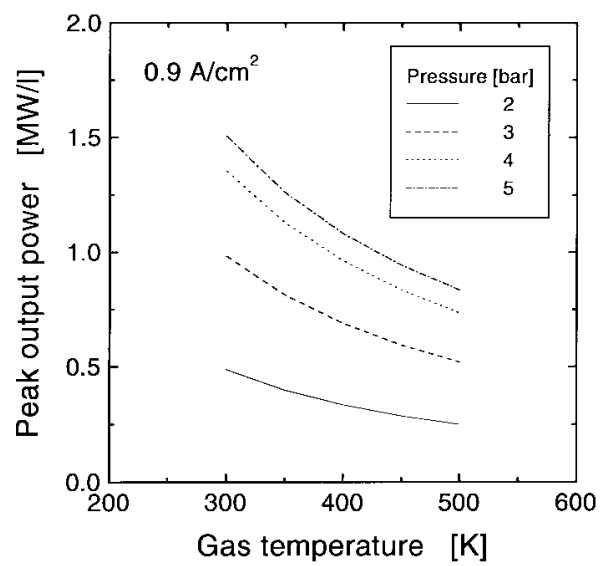

Fig. 13. Here, the peak output power is plotted versus the gas temperature for various pressures. Unlike Fig. 14, the drop of the peak output power is limited to about $30 \%$.

and a subsequent decrease of the output by electron collision mixing according to (16). In Fig. 12(a) and (b), the laser output power density and electron density versus the input power density are plotted for three different gas temperatures at a pressure of 5 bar and an $e$-beam current density of $0.9 \mathrm{~A} / \mathrm{cm}^{2}$. These graphs show that, although the increase of $n_{e}$ due to the temperature is relatively small, about $15 \%$ for a temperature difference of $100 \mathrm{~K}$, the decrease of the output power can be dramatic at high input powers. In Fig. 13, the peak value of the output power for several pressures is shown as a function of the gas temperature. We see that when the temperature increases from $300-500 \mathrm{~K}$ the peak value drops about $30 \%$. Because the heat capacity is proportional to the gas density, it is expected that the higher the gas pressure is the larger the stationary period at a fixed power deposition, which is in agreement with Fig. 3.

The temperature for which the laser may operate without degraded performance is also a strong function of the $e$ beam current density, which is related to the dependence of the electron energy on the e-beam current as mentioned before. This is shown for a beam current density of 0.4 and $0.9 \mathrm{~A} / \mathrm{cm}^{2}$ in Fig. 14(a) and (b), respectively. Here, the final gas temperature reached at the end of the stationary period, calculated from the dissipated discharge and beam energy, is plotted versus the total input power during that period. Thus this figure shows to what extent the gas temperature may be increased at a certain input power level before degradation of the performance occurs. It is seen that the lower the electron density produced by the discharge, the less the increased temperature effects the quenching and consequently the output. At low input powers, however, the duration of the stationary period is merely limited by our pump duration of $20 \mu \mathrm{s}$ and the indicated operating temperature is not the maximum allowable temperature that corresponds with that input power.

The maximum pulse energy defined as the energy during the stationary period depends not only on the gas pressure and power deposition, as we already described in the previous section, but also on the e-beam current. According to Fig. 7, this maximum is about 10 and $16 \mathrm{~J} / \ell$ for $e$-beam current densities of 0.4 and $0.9 \mathrm{~A} / \mathrm{cm}^{2}$, respectively. It should be noted 


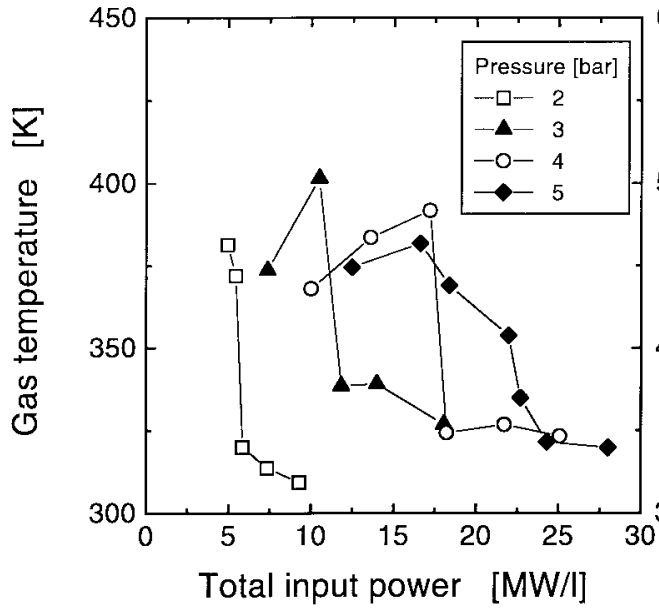

(a)

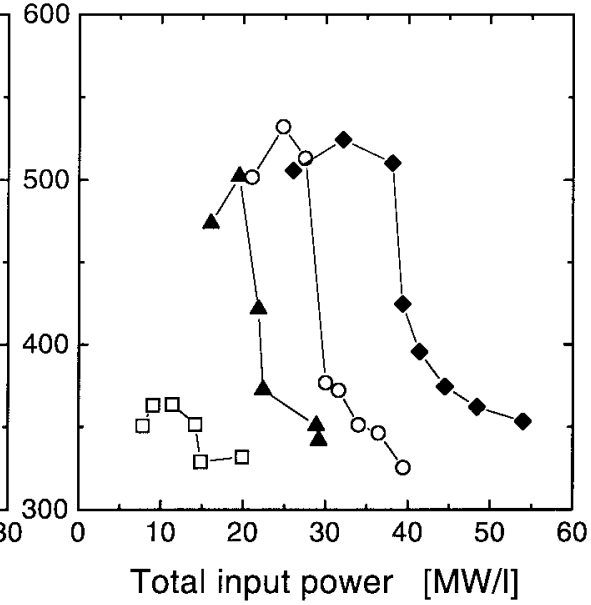

(b)

Fig. 14. The calculated gas temperature, at the point where the laser output starts to drop, as a function of the total input power for several pressures.

that if the total energy of a shot also includes the degraded part after the stationary period, the maximum total energy obtained in these experiments was $22 \mathrm{~J} / \ell$ at 5 bar. These results are considerably higher than our previous results obtained with an $e$-beam pulse of only about $1 \mu$ s at the same conditions [7].

Fig. 14 also shows that the increase of the $e$-beam current density, which has a favorable effect on lowering the electron quenching, allows an increase of the gas temperature before degradation starts. For example, at a beam current density of $0.4 \mathrm{~A} / \mathrm{cm}^{2}$ at $5 \mathrm{bar}$, the maximum temperature increase at the end of the stationary period is about $25 \mathrm{~K}$ for an input power of $25 \mathrm{MW} / \ell$, whereas a density of $0.9 \mathrm{~A} / \mathrm{cm}^{2}$ permits an increase of more than $200 \mathrm{~K}$ at the same power deposition.

It is noted that in experiments with fission fragment pumping Hebner [14] also found a dramatic drop of the output power when the temperature increased beyond approximately $400 \mathrm{~K}$. In his experiments, the output power was insensitive to the gas temperature below $400 \mathrm{~K}$.

\section{CONCLUSION}

The experiments have revealed a stationary duration of the output power that depends on the discharge power and gas pressure and not so much on the $e$-beam current. After the stationary region the efficiency drops. The output energy per pulse for which the efficiency is at optimum is then equal to the output power times the stationary duration. These values are plotted for different pressure in Fig. 7(a) and (b) as a function of the discharge power. The efficiencies for the discharge and total input power are plotted in, respectively, Figs. 5 and 6. We notice that the highest overall efficiency of about $8 \%$ is obtained for low pressure and low discharge power. The maximum discharge efficiency of $5 \%-6 \%$ is not so sensitive to the input power, especially at high pressure. Looking for the optimized output energy we find, according to Fig. 7, the best result for a gas pressure of 4 bar and an input discharge power of about $8 \mathrm{MW} / \ell$. The output power will then have a duration of $12 \mu \mathrm{s}$ at an $e$-beam current of $0.4 \mathrm{~A} / \mathrm{cm}^{2}$ and $16 \mu \mathrm{s}$ at an $e$-beam current of $0.9 \mathrm{~A} / \mathrm{cm}^{2}$. The total efficiency and output energies at these performances are, respectively, $5 \%$ at
$10 \mathrm{~J} / \ell$ and $4 \%$ at $16 \mathrm{~J} / \ell$. Depending on the repetition rate and gas volume, high average output powers above $100 \mathrm{~W}$ are feasible.

\section{ACKNOWLEDGMENT}

The authors wish to thank A. P. Napartovich and I. V. Kochetov for their helpful discussions and calculations and N. N. Ustinovskii for his useful suggestions.

\section{REFERENCES}

[1] N. G. Basov, V. A. Danylichev, A. Y. Dudin, D. A. Zayarnyi, N. N. Ustinovskii, I. V. Kholin, and A. Y. Chugunov, "Electron-beamcontrolled atomic Xe infrared laser," Sov. J. Quantum Electron., vol. 14, pp. 1158-1167, 1984.

[2] M. Ohwa, T. J. Moratz, and M. J. Kushner, "Excitation mechanisms of the electron-beam-pumped atomic xenon $(5 d \rightarrow 6 p)$ laser in $\mathrm{Ar} / \mathrm{Xe}$ mixtures," J. Appl. Phys., vol. 66, pp. 5131-5145, 1989.

[3] A. Suda, B. L. Wexler, K. J. Riley, and B. J. Feldman, "Characteristics of the high-pressure Ar-Xe laser pumped by an electron beam and an electron-beam sustained discharge," IEEE J. Quantum Electron., vol. 26, pp. 911-921, 1990.

[4] L. N. Litzenberger, D. W. Trainor, and M. W. McGeoch, "A $650 \mathrm{~J}$ e-beam-pumped atomic xenon laser," IEEE J. Quantum Electron., vol. 26, pp. $1668-1675,1990$.

[5] R. L. Watterson and J. H. Jacob, "Measurements of intrinsic efficiency and parameters of an electron beam pumped ArXe laser," IEEE $J$. Quantum Electron., vol. 26, pp. 417-422, 1990.

[6] H. Botma, P. J. M. Peters, and W. J. Witteman, "Intrinsic efficiency and critical power deposition in the e-beam sustained Ar:Xe laser," Appl. Phys. B, vol. 52, pp. 277-280, 1991.

[7] H. Botma, P. J. M. Peters, and W. J. Witteman, "Saturation studies of the e-beam sustained discharge atomic xenon laser," IEEE J. Quantum Electron., vol. 29, pp. 2519-2524, 1993.

[8] Y. B. Udalov, P. J. M. Peters, M. B. Heeman-Ilieva, F. H. J. Ernst, V. N. Ochkin, and W. J. Witteman, "New continuous wave infrared Ar-Xe laser at intermediate gas pressures pumped by a transverse radio frequency discharge," Appl. Phys. Lett., vol. 63, pp. 721-722, 1993.

[9] S. N. Tskhai, Y. B. Udalov, P. J. M. Peters, W. J. Witteman, and V. N. Ochkin, "Continuous wave near-infrared atomic Xe laser excited by a radio frequency discharge in a slab geometry," Appl. Phys. Lett., vol. 66, pp. 801-803, 1995.

[10] S. W. A. Gielkens, P. J. M. Peters, W. J. Witteman, P. V. Borovikov, A. V. Stepanov, V. N. Tskhai, M. A. Zavjalov, V. I. Gushenets, and N. N. Koval, "A long-pulse $300 \mathrm{keV}$ gun with a plasma cathode for high-pressure gas lasers," Rev. Sci. Instrum., vol. 65, pp. 2449-2452, 1996.

[11] J. J. Berger and S. M. Seltzer, Nucl. Sci. Ser. no. 10, NAS-NRC pub. 113, 1964. 
[12] T. J. Moratz, T. D. Saunders, and M. J. Kushner, "High-temperature kinetics in He and Ne buffered XeF lasers: The effect on absorption," Appl. Phys. Lett., vol. 54, pp. 102-104, 1989.

[13] A. P. Hickman, D. L. Huestis, and R. P. Saxon, "Interatomic potentials for excited states of XeHe and XeAr," J. Chem. Phys., vol. 96, pp. 2099-2113, 1992

[14] G. A. Hebner, "Gas temperature dependent output of the atomic argon and xenon lasers," IEEE J. Quantum Electron., vol. 31, 1626-1631, 1995.

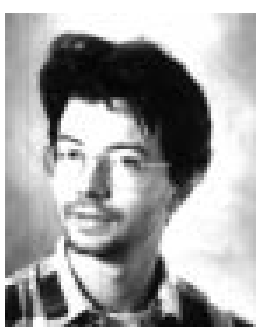

S. W. A. Gielkens was born in Heerlen, The Netherlands, on May 2, 1969. He received the M.Sc. degree from the University of Utrecht, The Netherlands, in 1993.

Since then, he has been working at the Quantum Electronics Group of the Department of Applied Physics at the University of Twente, Enschede, The Netherlands, on the high-pressure atomic Xe laser. His interests include gas-laser excitation mechanisms, laser kinetics, and laser spectroscopy.

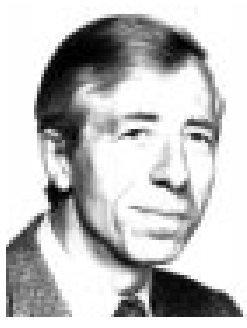

W. J. Witteman was born in Monster, The Netherlands, on December 12, 1933. He received the degree of mechanical engineer from the Technical University of Delft, The Netherlands, in 1958 and the Ph.D. degree in physics from the Technical University of Eindhoven, Eindhoven, The Netherlands, in 1963.

From 1958 to 1960 , he was first a Post-Doctoral Fellow and later a Research Associate at the Institute of Fluid Dynamics and Applied Mathematics, University of Maryland, College Park, studying molecular relaxation phenomena by means of shock-tube experiments. From 1961 until 1969, he was with Philips Research Laboratories, Eindhoven, The Netherlands, where he was engaged with high-pressure physics and technology until 1963. After that he did research on water-vapor lasers, sealed-off $\mathrm{CO}_{2}$ lasers, and argon-ion lasers. Since 1969, he has been a professor at the University of Twente, Enschede, The Netherlands, where he works on highpower pulsed laser systems. He is actively engaged in the field of $\mathrm{CO}_{2}$ lasers, mode-locking techniques, electron-beam and discharge-pulsed excimer lasers, both continuous and pulsed $\mathrm{CO}$ lasers, electro-ionization lasers like $\mathrm{Ar}-\mathrm{Xe}$ waveguide lasers, and free-electron lasers of the Raman and Compton type. Since 1989, he has been a managing director of the Nederlands Centrum voor Laser Research (NCLR) B.V., which is a joint venture between the University of Twente and the industry. The NCLR develops advanced laser systems for industrial applications, such as a $1-\mathrm{kW}$ Eureka-excimer laser operating at 1 $\mathrm{kHz}$.
V. N. Tskhai, photograph and biography not available at the time of publication.

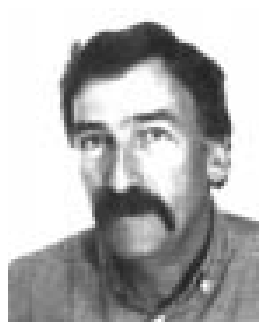

P. J. M. Peters was born in Meerlo, The Netherlands, on November 5, 1945. He received the M.Sc. degree from the Catholic University, Nijmegen, The Netherlands, and the Ph.D. degree in laser physics from the University of Twente, Enschede, The Netherlands, in 1981.

He then joined the staff of the Quantum Electronics Group of the Department of Applied Physics at the University of Twente where he is now an Associate Professor. He has carried out research mainly in the field of gas lasers. His Ph.D. work on a pulsed TEA CO laser was followed by research work on different types of excimer lasers, such as $\mathrm{KrF}, \operatorname{ArF}, \mathrm{XeF}(\mathrm{C}->\mathrm{A}$ and $\mathrm{B}->\mathrm{X})$, and on ionic excimers. Currently, he is engaged in research on the vacuum ultraviolet molecular F2 laser and in rare-gas recombination lasers such as the atomic Xe laser emitting in the near infrared. 